\title{
On the Consistency of Perturbativity and Gauge Coupling Unification
}

\author{
Joachim Kopp ${ }^{\mathrm{a}}$, Manfred Lindner ${ }^{\mathrm{b}}$, Viviana Niroc ${ }^{\mathrm{c}}$, Thomas E. J. Underwood ${ }^{\mathrm{d}}$ \\ Max-Planck-Institut für Kernphysik, \\ Postfach 1039 80, 69029 Heidelberg, Germany
}

September 16, 2009

\begin{abstract}
We investigate constraints that the requirements of perturbativity and gauge coupling unification impose on extensions of the Standard Model and of the MSSM. In particular, we discuss the renormalization group running in several SUSY left-right symmetric and Pati-Salam models and show how the various scales appearing in these models have to be chosen in order to achieve unification. We find that unification in the considered models occurs typically at scales below $M_{\not B}^{\min }=10^{16} \mathrm{GeV}$, implying potential conflicts with the non-observation of proton decay. We emphasize that extending the particle content of a model in order to push the GUT scale higher or to achieve unification in the first place will very often lead to non-perturbative evolution. We generalize this observation to arbitrary extensions of the Standard Model and of the MSSM and show that the requirement of perturbativity up to $M_{\not B}^{\min }$, if considered a valid guideline for model building, severely limits the particle content of any such model, especially in the supersymmetric case. However, we also discuss several mechanisms to circumvent perturbativity and proton decay issues, for example in certain classes of extra dimensional models.
\end{abstract}

\section{Introduction}

Among the many interesting open problems in particle physics is the question whether the Standard Model (SM) gauge coupling constants unify at some high energy scale. Even though such Grand Unification does not occur in the SM, one of its best motivated extensions, namely the Minimal Supersymmetric Standard Model (MSSM), does predict Grand Unification at $M_{\mathrm{GUT}} \simeq 10^{16} \mathrm{GeV}$ [1], opening up the possibility to embed the model into a Grand Unified Theory (GUT) based, for example, on the gauge group $S O(10)$.

Grand Unification is also possible in more complex models: Amaldi et al. have identified several extensions of the SM or MSSM particle content that would lead to gauge coupling unification [1, 2], and Lindner and Weiser have performed a similar study in left-right

\footnotetext{
${ }^{\text {a}}$ Email: jkopp@mpi-hd.mpg.de

${ }^{\mathrm{b}}$ Email: lindner@mpi-hd.mpg.de

${ }^{\mathrm{c}}$ Email: niro@mpi-hd.mpg.de

dEmail: Thomas.E.J.Underwood@mpi-hd.mpg.de
} 
symmetric models [3]. Recently, Calibbi et al. have derived a set of "magic fields" that can be added to the MSSM without spoiling unification [4]. Model-dependent studies have been carried out for example by Shaban and Stirling [5] for a non-SUSY left-right symmetric model and by Perez-Lorenzana and Mohapatra for models with extra dimensions [6]. A model independent study of non-SUSY left-right symmetric models has also been carried out by Perez-Lorenzana et al. [7]. Recently Aranda et al. carried out a study of extended Higgs sectors in SUSY GUTs, paying attention to the constraints coming from the requirements of perturbativity and freedom from anomalies [8].

Our aim in this work is to emphasize that the often adopted requirements that (1) all gauge couplings remain perturbative up to the GUT scale $M_{\mathrm{GUT}}$ and (2) $M_{\mathrm{GUT}}$ is large enough to suppress proton decay beyond the experimental limit severely constrain extensions of the SM or the MSSM. Even though non-perturbativity is not a principle problem but only a practical one, and proton decay can be suppressed even for low $M_{\mathrm{GUT}}$ in suitably constructed GUTs, we will in this paper accept both (1) and (2) as valid guidelines for model building, and investigate in detail the constraints that a model has to fulfil in order to be compatible with them.

In sec. 2, we will begin by introducing the formalism of RGEs in order to fix our notation. We will then proceed to a detailed investigation of Grand Unification in left-right symmetric and Pati-Salam models in secs. 3 and 4, respectively. There, we will discuss how the various energy scales appearing in these models are constrained by the requirement of successful perturbative unification compatible with proton decay bounds. In sec. 5, we will generalize our results, and discuss perturbativity issues in arbitrary extensions of the SM and the MSSM. Finally, in sec. [6 we will outline how perturbativity and proton decay constraints can be circumvented by more elaborate model building constructs such as extra dimension. We will summarize our results and conclude in sec. 7 .

\section{Renormalization group evolution of gauge coupling constants}

The dependence of the gauge coupling constants $g_{i}$ on the energy scale $\mu$ for a theory with gauge group $G=\prod_{i} G_{i}$ is given at one-loop order by

$$
16 \pi^{2} \frac{d g_{i}(t)}{d t}=b_{i}\left[g_{i}(t)\right]^{3}
$$

where $t=\ln \left(\mu / \mu_{0}\right)$ and $\mu_{0}$ is an arbitrary renormalization scale. The coefficients $b_{i}$, which are determined by the particle content of the model, have for non-supersymmetric models the form [9]

$$
b_{i}=\sum_{R} s(R) T_{i}(R)-\frac{11}{3} C_{2 i} . \quad \text { (non-SUSY models) }
$$

Here, the sum runs over all representations of the gauge group factor $G_{i}$, counted according to their multiplicity in the model. For example, in the Standard Model (SM) with its six left-handed quarks and six right-handed quarks, the 3-dimensional representation of $S U(3)_{c}$ has a multiplicity of 12 . The Dynkin index $T_{i}(R)$ of the representation $R$ of $G_{i}$ is defined by $\operatorname{tr}\left[t_{i}^{a}(R) t_{i}^{b}(R)\right]=\delta^{a b} T_{i}(R)$, with $t_{i}^{a}(R)$ being the generators of $G_{i}$ in the representation $R$. If 
$G_{i}=U(1), T_{i}(R)=[q(R)]^{2}$, where $q(R)$ is the charge corresponding to the representation $R$. The coefficient $s(R)$ has the value $2 / 3$ if $R$ is a multiplet of chiral fermions, while $c(R)=1 / 3$ if $R$ is a multiplet of complex scalars. Finally, $C_{2 i}$ is the quadratic Casimir operator of the adjoint representation of $G_{i}$. For supersymmetric models, eq. (2) has to be replaced by

$$
b_{i}=\sum_{R} T_{i}(R)-3 C_{2 i} . \quad \text { (SUSY models) }
$$

The solution of the one-loop renormalization group equation (11) can be written in the form

$$
\alpha_{i}^{-1}(t)=\alpha_{i}^{-1}\left(t_{0}\right)-\frac{1}{2 \pi} b_{i}\left(t-t_{0}\right)
$$

where $\alpha_{i}=\left[g_{i}(t)\right]^{2} / 4 \pi$.

An important observation and one of the central points of this paper is that adding new (non-singlet) matter particles to a given model will always increase at least one of the $b_{i}$, and hence will lead to larger values for the corresponding $\alpha_{i}(t)$ at $t>t_{0}$. For sufficiently large particle content, $\alpha_{i}(t)$ will reach the non-perturbative regime at relatively low scales.

All results presented in this paper will be based on the above one-loop RGEs. In the case of weak couplings this approximation is certainly justified, but when approaching the nonperturbative regime, $\alpha_{i}^{-1} \lesssim 1$, higher order effects will become relevant. Nevertheless, in the context of our study it is sufficient to define the non-perturbativity scale as the scale at which the one-loop approximations breaks down or, more specifically, as the scale at which the one-loop value of at least one of the $\alpha_{i}^{-1}$ becomes negative. (Of course, the physical $\alpha_{i}^{-1}$ will always remain positive, and it is just the invalidity of the one-loop approximation that can lead to negative values.)

Also, note that we neglect threshold corrections, which lead to an uncertainty of typically one order of magnitude for the non-perturbativity scale and the GUT scale.

\section{Grand Unification in Left-Right Symmetric Models}

We now illustrate the constraints that perturbativity and unification place on models with large particle content by considering three different left-right (LR) symmetric extensions of the Standard Model: (i) A non-supersymmetric model with Higgs triplets [10, 11, 12, 13], (ii) the "minimal" SUSY LR model [14], and (iii) a slightly extended SUSY LR model [15, 16. All three models have in common that quarks and leptons reside in the following representations under $S U(3)_{c} \times S U(2)_{L} \times S U(2)_{R} \times U(1)_{B-L}$ :

$$
\begin{aligned}
Q\left(3,2,1, \frac{1}{3}\right) & =\left(\begin{array}{c}
u \\
d
\end{array}\right) & Q^{c}\left(3^{*}, 1,2,-\frac{1}{3}\right) & =\left(\begin{array}{c}
d^{c} \\
-u^{c}
\end{array}\right) \\
L(1,2,1,-1) & =\left(\begin{array}{c}
\nu_{e} \\
e
\end{array}\right) & L^{c}(1,1,2,1) & =\left(\begin{array}{c}
e \\
-\nu_{e}
\end{array}\right) .
\end{aligned}
$$

(i) Non-SUSY LR model with triplet Higgs [10, 11, 12, 13]. In the non-supersymmetric case, the LR symmetry is broken down to the Standard Model at a scale $M_{\mathrm{LR}}$ by Higgs triplets

$$
\Delta(1,3,1,2) \quad \text { and } \quad \Delta^{c}(1,1,3,-2) .
$$


The second of these acquires a vev of order $M_{\mathrm{LR}}$ and thus breaks $S U(2)_{R} \times U(1)_{B-L}$ down to $U(1)_{Y}$, while the first one is required only to keep the particle content left-right symmetric. Fermion masses are generated by a Higgs bidoublet

$$
\Phi(1,2,2,0)
$$

with a vev of the order of the electroweak scale. Even though we do not need to worry about the details of the symmetry breaking mechanism in order to study the renormalization group evolution of the model, it is crucial to know the mass scales of all particles. A detailed investigation [13] shows that all Higgs particles in the model have masses of the order of $M_{\mathrm{LR}}$, except for an $S U(2)_{L}$ doublet emerging from the bidoublet $\Phi$ and playing the role of the SM Higgs boson. Note that, even though the model is generically non-supersymmetric, a high scale supersymmetrization at a scale $M_{\mathrm{SUSY}}>M_{\mathrm{LR}}$ is imaginable.

(ii) Minimal SUSY LR model [14]. The Higgs sector of this model is given by that of the non-SUSY model (i) (with all fields promoted to superfields), supplemented by two additional triplets

$$
\bar{\Delta}(1,3,1,-2) \quad \text { and } \quad \bar{\Delta}^{c}(1,1,3,2)
$$

required for anomaly cancellation and a singlet

$$
S(1,1,1,0)
$$

to ensure charge and $R$-parity conservation. Moreover, in the SUSY case two Higgs bidoublets $\Phi_{1}$ and $\Phi_{2}$ are required to allow for non-vanishing quark and lepton mixing angles. Of these Higgs superfields, four doublets as well as the doubly charged components of $\Delta^{c}$ and $\bar{\Delta}^{c}$ are light and have masses of $\mathcal{O}\left(M_{\mathrm{SUSY}}\right)$. In this study, we will make the simplifying assumption that these fields all have the same mass, $M_{\mathrm{SUSY}}$, whereas in practice, for low values of $M_{\mathrm{SUSY}}$ some of the fields are required to have slightly higher masses to satisfy constraints on, for example, flavour changing neutral currents. We have checked this simplification has a very minimal effect on our results.

(iii) Non-minimal SUSY LR model [15, 10]. The particle content of the non-minimal model is similar to that of the minimal SUSY-LR model, with the singlet $S$ being replaced by two triplets

$$
\Omega(1,3,1,0) \quad \text { and } \quad \Omega^{c}(1,1,3,0)
$$

uncharged under $U(1)_{B-L}$. Breaking of the left-right symmetry proceeds in two steps in this model:

$$
S U(2)_{R} \times U(1)_{B-L} \stackrel{M_{\mathrm{LR}}}{\longrightarrow} U(1)_{R} \times U(1)_{B-L} \stackrel{M_{B-L}}{\longrightarrow} U(1)_{Y} .
$$

The set of light particles includes the usual MSSM Higgses at the electroweak scale, the neutral components of $\Delta^{c}$ and $\bar{\Delta}^{c}$ at $M_{B-L}$, and the $\Omega$ field at $\max \left(M_{B-L}^{2} / M_{\mathrm{LR}}, M_{\mathrm{SUSY}}\right)$ [16].

In fig. 1 we compare the one-loop renormalization group running of the three considered models and of the MSSM. The embedding of the SM or MSSM into the standard GUT groups $S U(5)$ and $S O(10)$ requires $\alpha_{3}\left(M_{\mathrm{GUT}}\right)=\alpha_{2}\left(M_{\mathrm{GUT}}\right)=\frac{20}{3} \alpha_{1}\left(M_{\mathrm{GUT}}\right)$, but for the graphical presentation we have absorbed the factor $\frac{20}{3}$ into the definition of $\alpha_{1}$, so that at 
$M_{\mathrm{GUT}}$ the curves for $\alpha_{1}, \alpha_{2}$, and $\alpha_{3}$ meet at one point. In the left-right models, the GUT normalization factor is $\frac{8}{3}$ instead of $\frac{20}{3}$. The matching condition for the GUT-normalized $U(1)$ coupling constants at $M_{\mathrm{LR}}$ reads for models (i) and (ii)

$$
\alpha_{1, \mathrm{LR}}\left(M_{\mathrm{LR}}\right)=\frac{2}{5} \frac{\alpha_{1, \mathrm{SM}}\left(M_{\mathrm{LR}}\right) \alpha_{2}\left(M_{\mathrm{LR}}\right)}{\alpha_{2}\left(M_{\mathrm{LR}}\right)-\frac{3}{5} \alpha_{1, \mathrm{SM}}\left(M_{\mathrm{LR}}\right)},
$$

where $\alpha_{1, \mathrm{LR}}$ is the $U(1)_{B-L}$ coupling constant, while $\alpha_{1, \mathrm{SM}}$ and $\alpha_{2}$ correspond to $U(1)_{Y}$ and $S U(2)_{L}$, respectively. In model (iii), a condition of the form (13) is imposed not at $M_{\mathrm{LR}}$ but at $M_{B-L}$, with $\alpha_{2}$ replaced by the $U(1)_{R}$ coupling constant.

Fig. 1 shows that in all three models, unification is possible, but, especially in case (ii), tends to occur at rather low scales, in possible conflict with bounds from proton decay. In fact, from dimensional analysis, we expect the proton lifetime to be

$$
\tau_{p} \sim \frac{M_{\mathrm{GUT}}^{4}}{m_{p}^{5}} .
$$

The bound $\tau_{p}>2.1 \cdot 10^{29}$ yrs [17] then implies $M_{\mathrm{GUT}} \geq M_{\mathbb{B}}^{\min } \equiv 10^{16} \mathrm{GeV}$. Therefore, models with $M_{\mathrm{GUT}}<M_{\mathbb{B}}^{\min }$ can be embedded into a Grand Unified Theory only if special measures are taken to forbid or suppress proton decay operators beyond the estimate (14). Note also that the unified coupling constant $\alpha_{\mathrm{GUT}}$ has a much larger value in the supersymmetric models than in the non-SUSY case. The reason is that according to eqs. (21) and (3) the additional particle content of SUSY models always increases the beta function coefficients $b_{i}$.

In general, in supersymmetric models, besides the (dimension-six) operators induced by $\mathrm{X}$ and $\mathrm{Y}$ gauge boson exchange, proton decay can be induced by additional dimensionfive operators arising from the exchange of coloured higgsinos. These are really dangerous operators, since they lead to extremely fast proton decay. Indeed, if they are present, the proton life-time $\tau_{p}$ becomes proportional only to the second power in the GUT scale, instead of the fourth power, as reported in eq. (14).

The purely supersymmetric contributions to proton decay have already been used to set limits on SUSY-GUT models. For example, the minimal supersymmetric $S U(5)$ GUT model has been tightly constrained by the Super-Kamiokande lower bound on $p \rightarrow K^{+} \bar{\nu}$ decay channel [18], assuming that the gauge coupling unification is satisfied. Several works have dealt with the possibility of suppressing dimension-five operators. Some of these models invoke extra-dimensions, see e.g. [19, 20] or a more complicated higgs sector [21, 22].

However, in our work we decided to pursue a conservative approach and thus apply only the constraint on the unification scale derived through eq. (14). It is, however, possible that some models with a unification scale $M_{\mathrm{GUT}} \gtrsim M_{\mathbb{B}}^{\text {min }}$ could be excluded by rapid proton decay induced by dimension-five operators ${ }^{1}$.

Let us now examine how varying the scales $M_{\mathrm{LR}}, M_{\mathrm{SUSY}}$, and $M_{B-L}$ affects the prospects of Grand Unification in left-right-symmetric models. For the non-SUSY model (i), only the

\footnotetext{
${ }^{1}$ In principle, beyond gauge boson and higgsino exchange, two other sources of proton decay can be present: R-parity violating terms and dimension-five Planck suppressed operators. However, these operators are not directly related to the unification scale (since they can also be present without unification), and therefore they do not provide a model-independent constraint on the value of $M_{\mathrm{GUT}}$.
} 


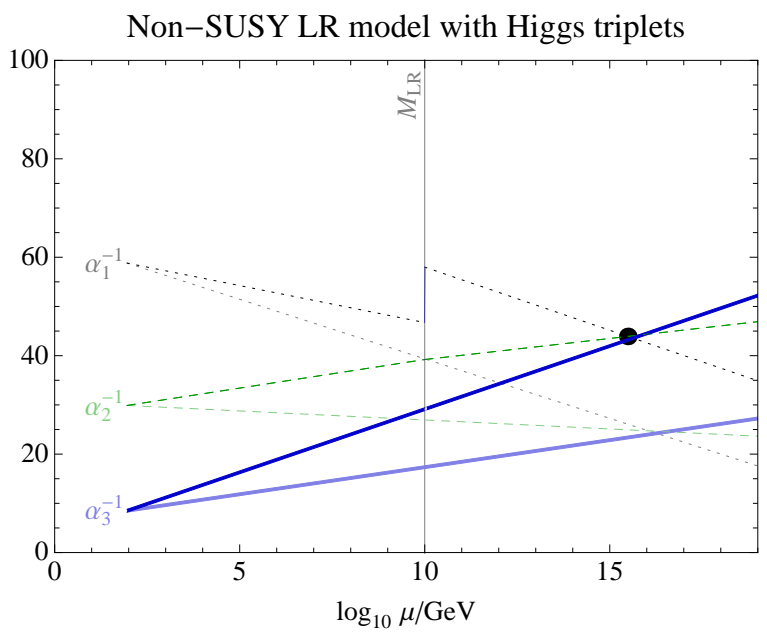

(i)

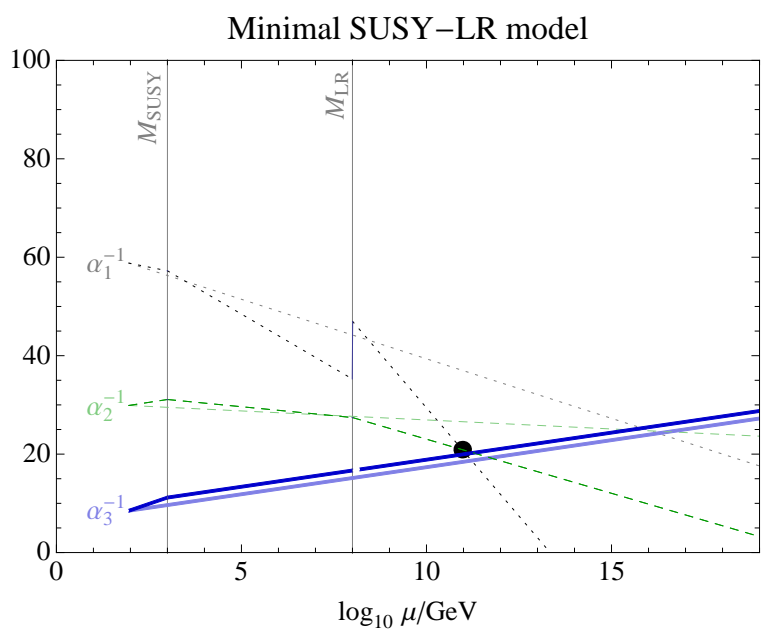

(ii)

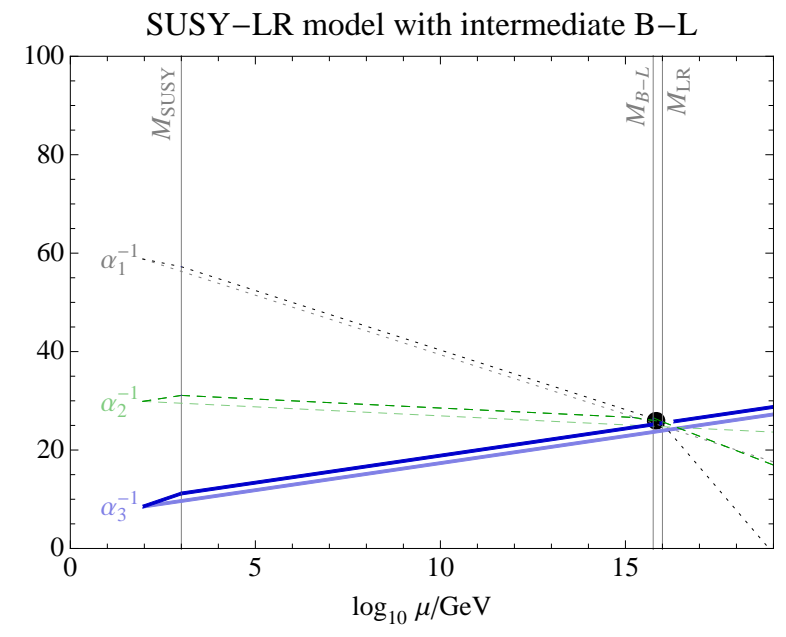

(iii)

Figure 1: Renormalization group evolution in (i) a non-supersymmetric left-right model [13], (ii) the minimal supersymmetric LR model [14], and (iii) a non-minimal SUSY-LR model [15, 16]. The light curves in the background correspond to the renormalization group running in the MSSM. 
choice $M_{\mathrm{LR}} \sim 10^{10} \mathrm{GeV}$ (shown in fig. 1) leads to unification. For the SUSY models, the indicated areas in fig. 2 show for which combinations of $M_{\mathrm{LR}}$ and $M_{\mathrm{SUSY}}$ unification occurs. (For model (iii) (right panel), for given values of $M_{\mathrm{LR}}$ and $M_{\mathrm{SUSY}}$, we chose the unique value $M_{B-L}$ in the range $\left[M_{\mathrm{SUSY}}, M_{\mathrm{LR}}\right]$ which leads to unification). The GUT scale is marked on each plot either explicitly along the line of values leading to unification (model (ii)), or through the shaded contours (model (iii)). Notice that in most of the parameter space unification is only possible in a narrow band of $M_{\mathrm{SUSY}}$ and $M_{\mathrm{LR}}$ values, and that in virtually all of these cases we find $M_{\mathrm{GUT}}<M_{\not B}^{\mathrm{min}}$, thus causing potential problems with proton decay.

Let us also remark that we have not found any solutions with $M_{\mathrm{GUT}} \sim M_{\mathrm{Pl}}$, which would have been an interesting feature in the context of quantum gravity theories. For model (iii), all unifying solutions we have found correspond to $M_{L R} \sim M_{\mathrm{GUT}}$. (This observation is similar to what has been found in [23] for a class of $S O(10)$ GUTs.)

One might hope to reconcile LR symmetry with Grand Unification above $M_{\not B}^{\min }$ (or even at $M_{\mathrm{Pl}}$ ) by extending the particle content of the LR model. In particular, the addition of extra coloured particles could postpone unification, but, as discussed above, any new particle will inevitably bring the model closer to non-perturbativity. The orange shaded regions in the upper panels of fig. 2 show for which values of $M_{\text {SUSY }}$ and $M_{\text {LR }}$ it is definitely impossible to reconcile unification, perturbativity and the proton decay bounds by adding extra matter because at least one of the $\alpha_{i}$ becomes non-perturbative below $M_{\not B}^{\text {min }}$, even without additional particles in the model. For model (iii) there is also a yellow region in which this happens only for some choices of $M_{B-L}$. In these regions of parameter space, any attempt to increase $M_{\mathrm{GUT}}$ by adding new scalar or fermionic particles would be in even greater conflict with perturbativity. We see that the problem is particularly severe in the minimal SUSY-LR model (ii). The reason is that this model has many particles with low masses around $M_{\mathrm{SUSY}}$. In particular, the doubly charged scalars $\delta^{c--}$ and $\bar{\delta}^{c++}$ have a very strong impact on the running of $\alpha_{1}$.

\section{Grand Unification in a SUSY Pati-Salam model}

Let us now investigate Grand Unification in another well-motivated class of models, namely those of the Pati-Salam (PS) type [24] with the gauge group $S U(2)_{L} \times S U(2)_{R} \times S U(4)$. In particular, we will study the minimal supersymmetric PS model discussed in [25]. The gauge symmetry breaking pattern of this model is

$$
\begin{aligned}
& S U(2)_{L} \times S U(2)_{R} \times S U(4) \\
& \quad \stackrel{M_{\mathrm{PS}}}{\longrightarrow} S U(3)_{c} \times S U(2)_{L} \times S U(2)_{R} \times U(1)_{B-L} \\
& \quad \stackrel{M_{\mathrm{LR}}}{\longrightarrow} S U(3)_{c} \times S U(2)_{L} \times U(1)_{Y},
\end{aligned}
$$

and SUSY is broken at $M_{\mathrm{SUSY}}<M_{\mathrm{LR}}$. The matter particles reside in the representations

$$
\psi(2,1,4) \quad \text { and } \quad \psi^{c}\left(1,2,4^{*}\right)
$$

of the PS gauge group. They get masses from the vevs of the Higgs bidoublets

$$
\Phi(2,2,1) \quad \text { and } \Phi(2,2,15) \text {. }
$$



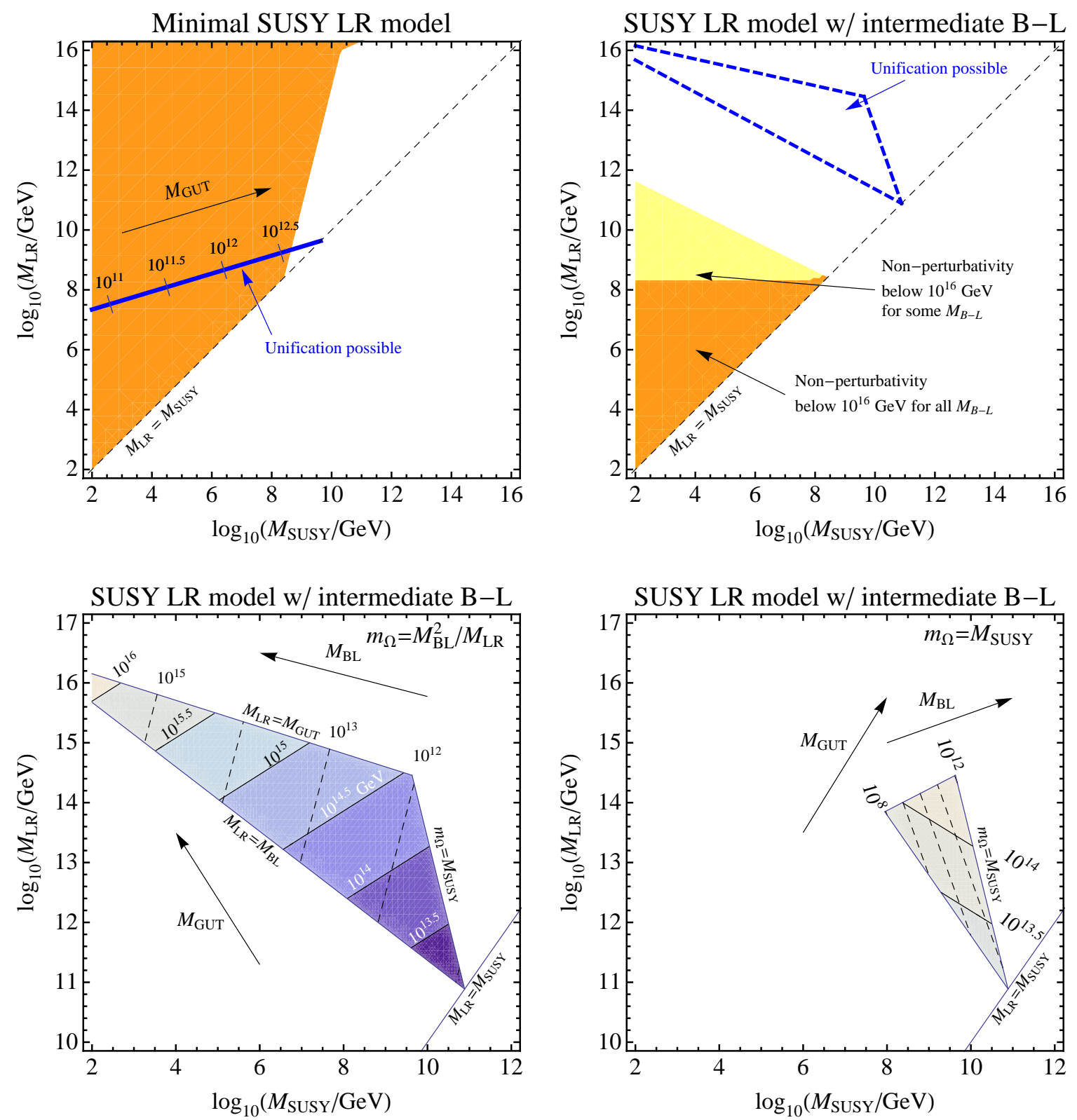

Figure 2: Gauge coupling unification and non-perturbativity constraints on left-right symmetric models. The regions of parameter space leading to successful unification are marked on each plot, along with the unification scale, $M_{\mathrm{GUT}}$. The dark orange areas depict combinations of $M_{\mathrm{SUSY}}$ and $M_{\mathrm{LR}}$ for which the model becomes non-perturbative below the proton decay scale, $M_{\not B}^{\min }$, so that even increasing the particle content at some intermediate scale will not be able to push $M_{\mathrm{GUT}}$ above $M_{\not B}^{\text {min }}$ without violating perturbativity. For the model with intermediate $B-L$ breaking we also show scenarios where non-perturbativity below $M_{\not B}^{\text {min }} \mathrm{GeV}$ occurs only for some choices of $M_{B-L}$ (light yellow area). In addition, the lower panels show the GUT scale (coloured shaded contours) and the $B-L$ breaking scale (dashed contours) required to achieve unification in this model. The lower left panel applies to the model where the mass of the $\mathrm{SU}(2)_{L}$ triplet, $m_{\Omega}=M_{B-L}^{2} / M_{L R}$ and the right panel applies to the case where $m_{\Omega}=M_{\mathrm{SUSY}}$. 


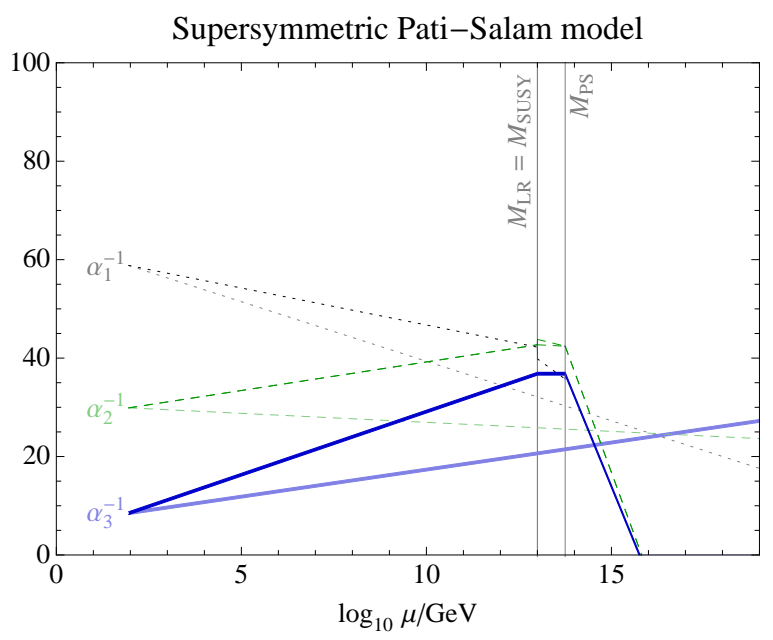

Figure 3: Renormalization group evolution in the minimal SUSY Pati-Salam model [25].

Symmetry breaking is achieved by introducing Higgs multiplets

$$
A(1,1,15)
$$

and

$$
\Sigma(3,1,10), \quad \bar{\Sigma}\left(3,1,10^{*}\right), \quad \Sigma^{c}\left(1,3,10^{*}\right), \quad \bar{\Sigma}^{c}(1,3,10)
$$

The particles surviving below $M_{\mathrm{PS}}$ are the usual matter particles, a colour octet with mass $M_{\mathrm{LR}}^{2} / M_{\mathrm{PS}}$ or $M_{\mathrm{SUSY}}$ (whichever is larger) emerging from $A$, and two $S U(2)_{R}$ triplets $\Delta^{c}(1,1,3,-2)$ and $\bar{\Delta}^{c}(1,1,3,2)$ of $S U(3)_{c} \times S U(2)_{L} \times S U(2)_{R} \times U(1)_{B-L}$. The doubly charged components of $\Delta^{c}$ and $\bar{\Delta}^{c}$ and a linear combination of their neutral components have masses of order $M_{\mathrm{SUSY}}$, while the remaining components have masses of order $M_{\mathrm{LR}}$.

An example for the running of the gauge couplings in the minimal SUSY PS model is shown in fig. 3. After a detailed investigation of the favoured values for $M_{\mathrm{PS}}, M_{\mathrm{LR}}$, and $M_{\text {SUSY }}$ we find that the Grand Unification of all gauge couplings is only possible in a very narrow region of parameter space, and even then only by being flexible in the uncertainty of $\alpha_{3}^{-1}\left(M_{Z}\right)$. For the example in fig. 3, Grand Unification is only possible for rather high values of $M_{\mathrm{SUSY}}$, implying that SUSY cannot be considered as a solution to the hierarchy problem here. Even partial unification into the Pati-Salam group $S U(2)_{L} \times S U(2)_{R} \times S U(4)$ is only possible for certain combinations of $M_{\mathrm{SUSY}}$ and $M_{L R}$, as shown in fig. 4. There, we also show the corresponding Pati-Salam scales $M_{P S}$ (shaded contours), as well as the scales at which the Pati-Salam coupling constants enter the non-perturbative regime (dotted lines). We see that the non-perturbativity scale is always below $M_{\not B}^{\min }$, implying that the minimal SUSY Pati-Salam model cannot be further unified without violating proton decay bounds (unless fundamentally new concepts such as extra dimensions are introduced, see sec. 6). 

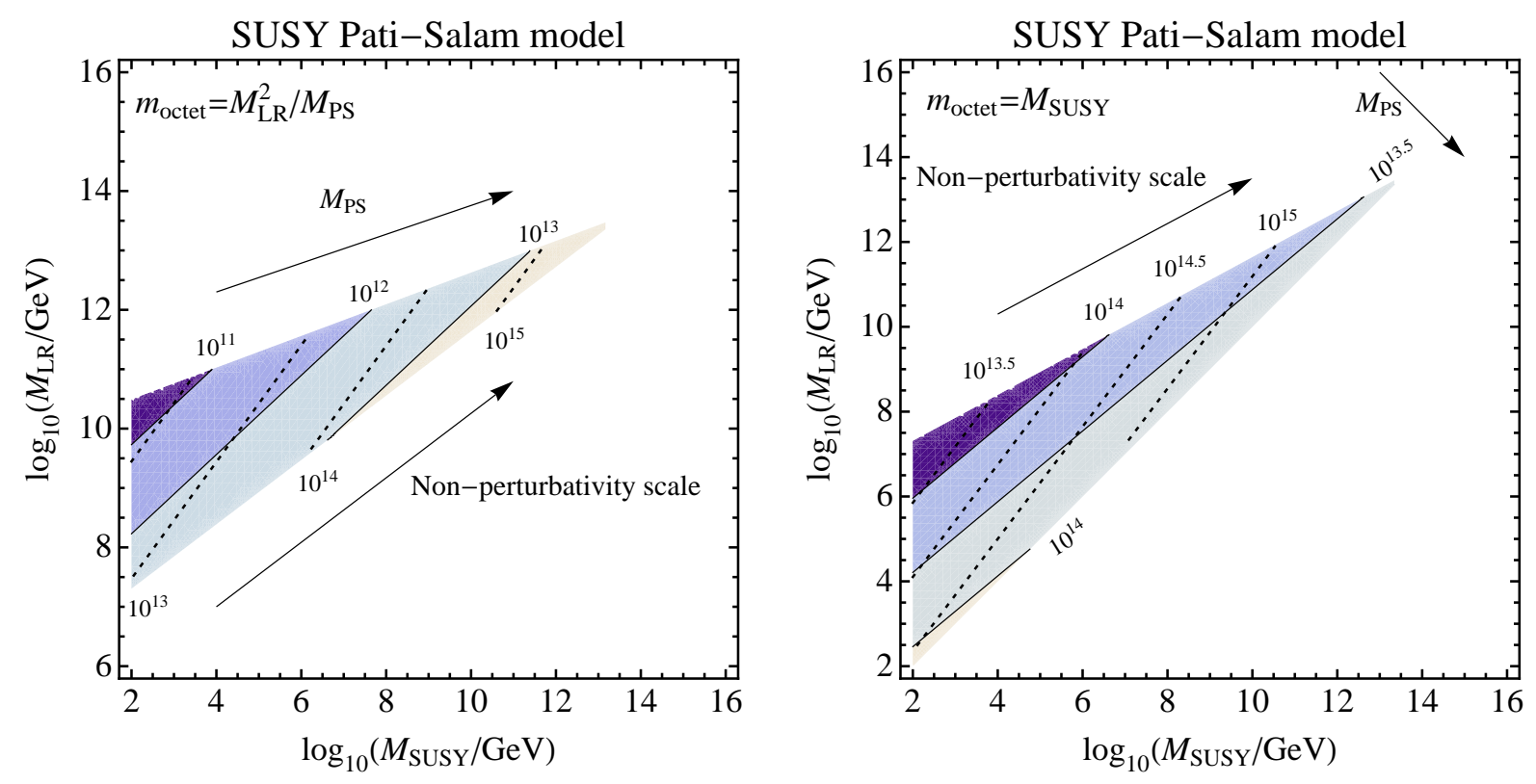

Figure 4: Constraints on the minimal SUSY Pati-Salam model [25] coming from successful, perturbative, unification into the Pati-Salam group. The shaded region shows the part of parameter space where Pati-Salam unification is possible and the shaded contours indicate the Pati-Salam scale. The scale at which the model becomes non-perturbative is illustrated by the dashed contours.

\section{Model-independent discussion of perturbativity and Grand Uni- fication}

Let us now generalize our findings from the previous sections to arbitrary extensions of the Standard Model. The observation that models with large particle content enter the non-perturbativity regime at relatively low scales is quite generic (for exceptions, see sec. 6) since it follows directly from the fact that additional matter particles always increase the coefficients $b_{i}$ (see equation (3)) Therefore, if perturbativity up to the GUT scale is demanded in such models, gauge coupling unification must also occur at relatively low scales, in tension with proton decay bounds that suggest $M_{\mathrm{GUT}} \gtrsim M_{\not B}^{\min } \sim 10^{16} \mathrm{GeV}$. Thus, models with large particle content are disfavoured over more economic ones.

To formulate the perturbativity constraints on models of new physics more quantitatively, let us assume an arbitrary extensions of the SM or MSSM particle content at a scale $\mu^{\text {new }}$, with the new particles giving contributions $b_{i}^{\text {new }}$ to the $\beta$-function coefficients $b_{i}$. The three panels of fig. 5 show the scales where $\alpha_{1}, \alpha_{2}$, and $\alpha_{3}$ become infinite in the one-loop approximation as a function of $\mu^{\text {new }}$ and $b_{i}^{\text {new }}$. Fig. 6 shows similar results for the MSSM. We see that for new physics at the TeV scale, an increase of $b_{1}$ by 8 or of $b_{2}$ or $b_{3}$ by 9 would render the SM non-perturbative below $M_{\not B}^{\mathrm{min}}$, while for the MSSM this would happen already if any of the $b_{i}^{\text {new }}$ becomes larger than 5 . If new particles are introduced well above the electroweak scale, the perturbativity constraints become weaker.

In table 1, we list the contributions to the $b_{i}^{\text {new }}$ for various hypothetical new particle 
representations. We see that especially large representations with high hypercharge are problematic: For example, $\alpha_{3}$ would become non-perturbative below $10^{16} \mathrm{GeV}$ if the SM is extended by three vector-like colour octet quarks at $1 \mathrm{TeV}$. One the other hand, adding Higgs doublets or triplets to the SM is not a problem: $\alpha_{2}$ remains perturbative up to the Planck scale even if 48 new Higgs doublets or 12 Higgs triplets are added at the electroweak scale. In the MSSM, there is slightly less freedom to extend the particle content: Perturbativity of $\alpha_{2}$ is lost below $10^{16} \mathrm{GeV}$ if 10 additional $S U(2)$ doublets or 3 triplets are added, and $\alpha_{1}$ would run to $\infty$ below $10^{16} \mathrm{GeV}$ if $9 Y=2$ superfields are introduced.

Many extensions of the SM focus especially on the electroweak sector, which has much more room for interesting new phenomena at high energy than the QCD sector. Therefore, we will now consider models with no new coloured particles, i.e. $b_{3}^{\text {new }}=0$, but with arbitrary contributions to $b_{1}$ and $b_{2}$ from particles at the TeV scale. For models of this type, we plot in fig. 7 the non-perturbativity scales for $\alpha_{1}$ and $\alpha_{2}$ (shaded areas), and we indicate those combinations of $b_{1}^{\text {new }}$ and $b_{2}^{\text {new }}$ that lead to (perturbative) gauge coupling unification (gray points). Dark points correspond to a high GUT scale, while lighter ones stand for low $M_{\mathrm{GUT}}$. We see that Grand Unification is possible in a certain band of $b_{1}^{\text {new }}$ and $b_{2}^{\text {new }}$ values. Of course, even in those cases where no gauge coupling unification has been found in our plot, it can be forced by adding suitably chosen coloured representations to the model, provided that the points where $\alpha_{1}^{-1}$ and $\alpha_{2}^{-1}$ meet lies below the SM/MSSM curve for $\alpha_{3}^{-1}$, but still in the perturbative regime. We can also read from fig. 7 that for large beta function coefficients the GUT scale is always rather low, in possible conflict with proton decay. Again, one might hope to increase $M_{\mathrm{GUT}}$ by adding more particles, especially coloured ones, but this will inevitably lead to non-perturbative evolution if the beta function coefficients become too large.

Thus we conclude that, for models in which the SM or the MSSM is extended only by additional matter fields or Higgs particles at the electroweak scale, Grand Unification above $M_{\not B}^{\text {min }}$ is only possible for $b_{1}^{\text {new }}$ and $b_{2}^{\text {new }}$ lying in the unshaded region of fig. 7. Even then,

in all cases except for the pure MSSM, pushing $M_{\mathrm{GUT}}$ above $M_{\mathbb{B}}^{\min }$ requires exotic coloured particles.

To end this section, we summarize the implications of perturbativity, unification, and proton decay constraints for model building in the flow chart shown in fig. 8. Perturbativity is particularly problematic in non-minimal SUSY models (scenarios (A2) and (B2)) because these tend to have very large particle content. If SUSY does not exist up to $M_{\mathrm{Pl}}$ (cases (C1) and (C2)), the perturbativity constraint can be fulfilled more easily, but we encounter other difficulties, in particular the hierarchy problem. Taking these considerations into account, the most attractive of the considered scenarios is SUSY in its minimal form - the MSSM (or, equivalently, the NMSSM, which differs from the MSSM only by the addition of one gauge singlet, see [26] and references therein). Since the MSSM does not provide gauge coupling unification and cannot solve the hierarchy problem if $M_{\mathrm{SUSY}} \gg M_{Z}$ (case (B1)), we are left with case (A1), the MSSM with $M_{\text {SUSY }}$ around the LHC scale.

\section{Circumventing the perturbativity constraints}

In the analysis carried out in the previous sections, two main problems with extensions of the Standard Model have appeared. 

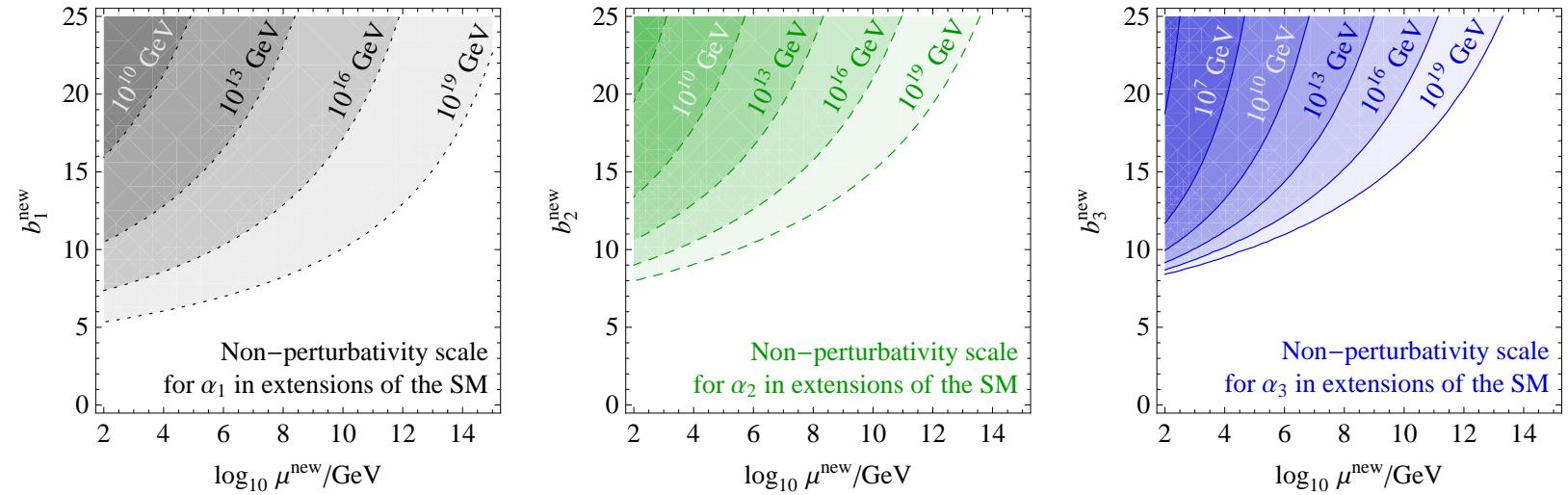

Figure 5: Perturbativity constraints on extensions of the SM. We assume that new scalar or fermionic particles with masses of order $\mu^{\text {new }}$ are introduced and give contributions $b_{i}^{\text {new }}$ to the $\beta$-function coefficients. The contours show the scale at which the one-loop approximations for the running coupling constants $\alpha_{i}$ become infinite as a function of $\mu^{\text {new }}$ and $b_{i}^{\text {new }}$.
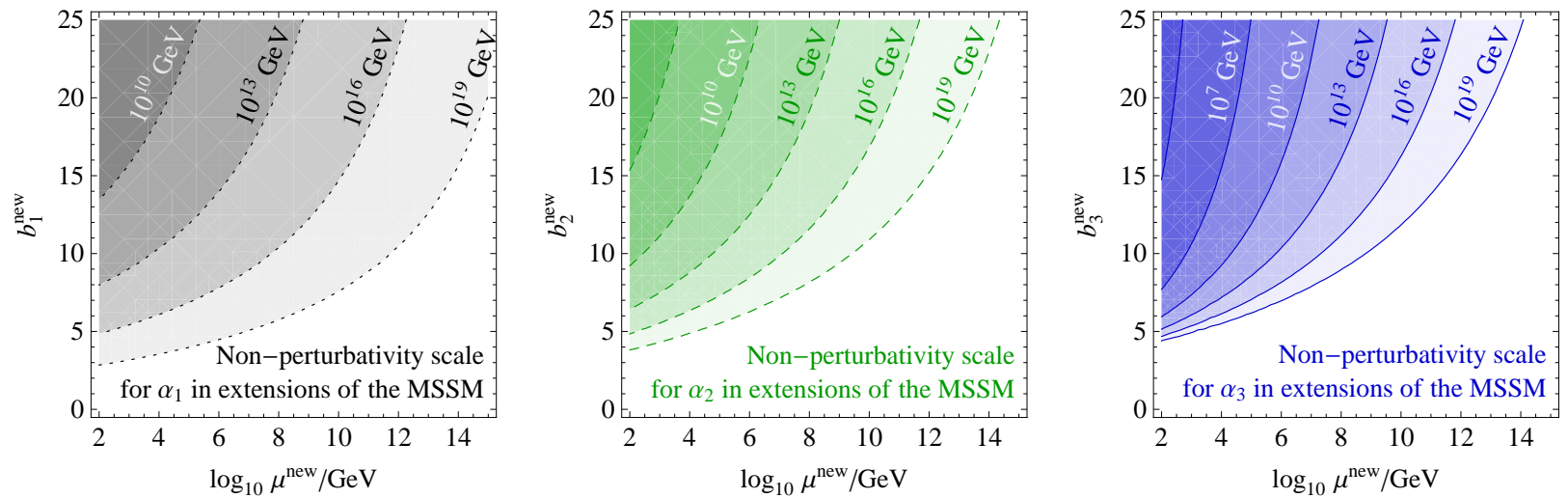

Figure 6: Similar to fig. 5, but for extensions of the MSSM with $M_{\mathrm{SUSY}} \sim 10^{2} \mathrm{GeV}$. 


\begin{tabular}{rccc}
\hline MSSM rep. & $b_{1}^{\text {new }}$ & $b_{2}^{\text {new }}$ & $b_{3}^{\text {new }}$ \\
\hline$(Y, 1,1)$ & $0.15 Y^{2}$ & 0 & 0 \\
$(Y, 2,1)$ & $0.3 Y^{2}$ & 0.5 & 0 \\
$(Y, 3,1)$ & $0.45 Y^{2}$ & 2 & 0 \\
$(Y, 4,1)$ & $0.6 Y^{2}$ & 5 & 0 \\
$(Y, 5,1)$ & $0.75 Y^{2}$ & 10 & 0 \\
$(Y, 6,1)$ & $0.9 Y^{2}$ & 17.5 & 0 \\
$(Y, 7,1)$ & $1.05 Y^{2}$ & 28 & 0 \\
$(Y, 1,3)$ & $0.45 Y^{2}$ & 0 & 0.5 \\
$(Y, 2,3)$ & $0.9 Y^{2}$ & 1.5 & 1 \\
$(Y, 3,3)$ & $1.35 Y^{2}$ & 6 & 1.5 \\
$(Y, 4,3)$ & $1.8 Y^{2}$ & 15 & 2 \\
$(Y, 5,3)$ & $2.25 Y^{2}$ & 30 & 2.5 \\
$(Y, 6,3)$ & $2.7 Y^{2}$ & 52.5 & 3 \\
$(Y, 7,3)$ & $3.15 Y^{2}$ & 84 & 3.5 \\
$(Y, 1,6)$ & $0.9 Y^{2}$ & 0 & 2.5 \\
$(Y, 2,6)$ & $1.8 Y^{2}$ & 3 & 5 \\
$(Y, 3,6)$ & $2.7 Y^{2}$ & 12 & 7.5 \\
$(Y, 4,6)$ & $3.6 Y^{2}$ & 30 & 10 \\
$(Y, 5,6)$ & $4.5 Y^{2}$ & 60 & 12.5 \\
$(Y, 6,6)$ & $5.4 Y^{2}$ & 105 & 15 \\
$(Y, 7,6)$ & $6.3 Y^{2}$ & 168 & 17.5 \\
$(Y, 1,8)$ & $1.2 Y^{2}$ & 0 & 3 \\
$(Y, 2,8)$ & $2.4 Y^{2}$ & 4 & 6 \\
$(Y, 3,8)$ & $3.6 Y^{2}$ & 16 & 9 \\
$(Y, 4,8)$ & $4.8 Y^{2}$ & 40 & 12 \\
$(Y, 5,8)$ & $6 Y^{2}$ & 80 & 15 \\
$(Y, 6,8)$ & $7.2 Y^{2}$ & 140 & 18 \\
$(Y, 7,8)$ & $8.4 Y^{2}$ & 224 & 21 \\
\hline & & &
\end{tabular}

Table 1: Contributions of hypothetical new particles to the $\beta$-function coefficients $b_{i}$. Numbers are given for chiral superfields; in the non-SUSY case, they have to be multiplied by $1 / 3$ for complex scalars and by $2 / 3$ for chiral fermions. Note that the values for $b_{1}$ include the GUT normalization factor $\frac{3}{20}$. 

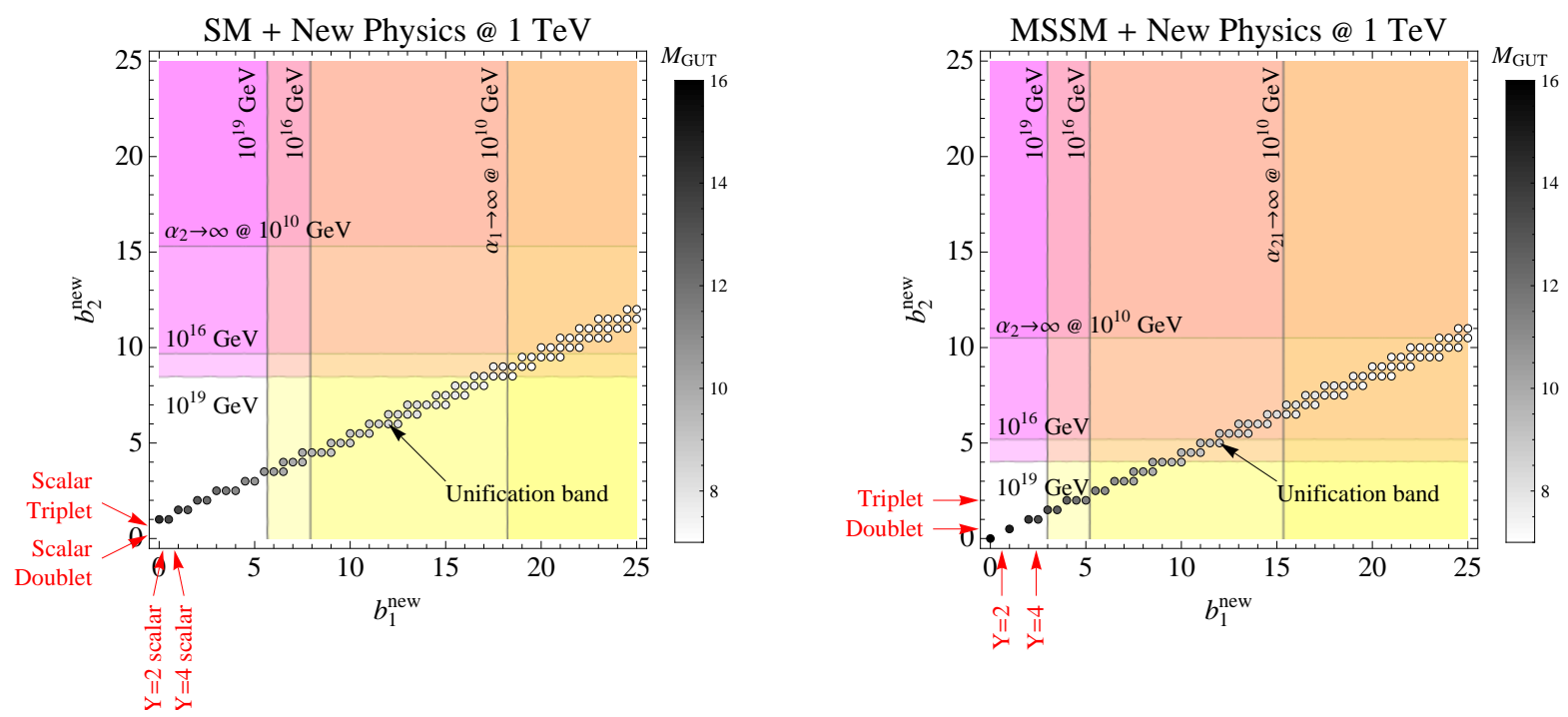

Figure 7: Perturbativity and Grand Unification in extensions of the electroweak sector of the SM (left panel) and the MSSM (right panel), assuming new physics at $1 \mathrm{TeV}$. The shaded areas indicate the scale where $\alpha_{1}$ and $\alpha_{2}$ become non-perturbative as a function of the new particles' contributions to the beta function coefficients, $b_{1}^{\text {new }}$ and $b_{\text {new }}^{2}$. The band of gray points shows for which combinations of $b_{1}^{\text {new }}$ and $b_{2}^{\text {new }}$ Grand Unification occurs. Dark points correspond to a high GUT scale, while lighter ones stand for low $M_{\mathrm{GUT}}$.

The first one is represented by the non-perturbative running of the gauge couplings at high scales when the particle content is increased with respect to the Standard Model or the MSSM. In figs. 5 and 6 we have quantified the limits that the new contributions to the $\beta$-function coefficients have to fulfil to preserve perturbative coupling constants $\alpha_{i}$.

In principle, we could also accept the divergent evolution of the coupling constants at high scales. However this means that we lose the ability to make predications about physics above the non-perturbativity scale and, moreover, we lose the ability to verify one of the main theoretical justifications for physics beyond the SM, i.e. gauge coupling unification.

A possible way out could be provided by embedding the gauge group of the model into a larger group at an intermediate scale $M_{I}$. This "partial unification" will change the matter contribution $T_{i}(R)$ as well as the gauge field contribution $C_{2 i}$ in eqs. (2) and (3) and could thus decrease $b_{i}$, keeping the model perturbative up to the Planck scale. Moreover, the matching conditions at $M_{I}$ could be such that the coupling of the new gauge group above $M_{I}$ is smaller than the corresponding coupling constants of the SM, in the same way as the $\alpha_{1}$ corresponding to $U(1)_{B-L}$ in the LR models can be smaller than the $\alpha_{1}$ corresponding to $U(1)_{Y}$ in the SM by virtue of eq. (13), see fig. 1 (i). We extensively investigate different classes of left-right models in sec. 3 and Pati-Salam models in sec. 4. Our study shows that also in scenarios with partial unification, one can easily run into similar perturbativity problems as in models that preserve the SM gauge group up to high scales. Similar difficulties are encountered in other models, for example the SUSY Little Higgs model considered in ref. [27] which encounters non-perturbativity below the unification scale when extra matter 


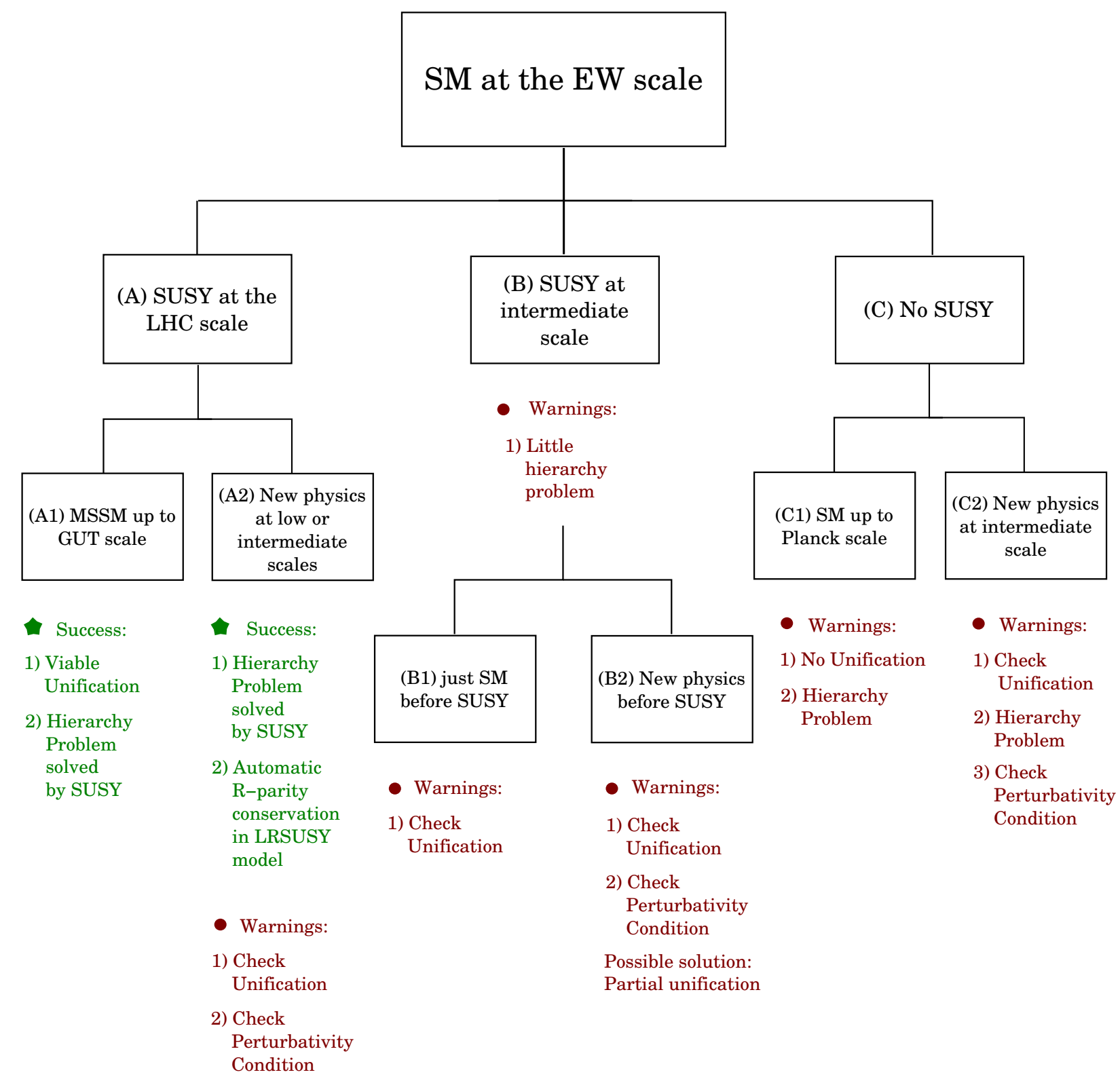

Figure 8: A "new physics" flow chart. 
is added to generate the top quark mass.

A realistic solution of the perturbativity problem is represented by extra-dimension models in which only the gauge fields propagate in the bulk, see e.g. [6]. In this case the number of gauge degrees of freedom increases thus also increasing the negative contributions to the $\beta$ function. The direct consequence is that the gauge couplings are pulled away from the Landau pole and their perturbative evolution can be followed up to the unification scale.

Low energy unification scales, $M_{\mathrm{GUT}}<M_{\not B}^{\mathrm{min}}$ represent the second problem that we experience in extensions of the Standard Model. Using the naive dimensional estimate of eq. (14), it can be found that such low values for $M_{\mathrm{GUT}}$ imply values of the proton lifetime $\tau_{p}$ that do not fulfil the experimental constraints provided by the Super-Kamiokande detector [28, 29]. However, the lower limit on the unification scale can be relaxed if proton decay is forbidden or sufficiently suppressed so that the proton lifetime $\tau_{p}$ becomes much larger than the estimate of eq. (14).

One possibility to, in part, evade these experimental proton decay bounds is to construct a "contorted flavours" GUT model [30]. Indeed, there is no a-priori reason to couple the first quark family with the first lepton family in a GUT multiplet. For example we could have the $(u, d)$ quarks in the same representation as the $\left(\nu_{\tau}, \tau\right)$ or $\left(\nu_{\mu}, \mu\right)$. It has been shown in [30] that this pattern can lead to the correct fermion masses and can kinematically suppress the proton decay channels into charged leptons. However, the decay channels involving neutrinos are still present and the experimental constraints reported in [17, 29] have to be taken into consideration. In this case, it is not possible to completely remove the constraints on the GUT scale arising from the experimental bounds on the proton lifetime.

An economical way to avoid the constraint on the GUT scale is achieved by extending the Higgs sector, see e.g. [31] for the case of an SU(5) GUT. In this way the baryon number violating mixing matrix is, in general, no longer related to the baryon conserving one and proton decay can be suppressed by correctly adjusting the mixing angle in the baryon number violating matrix. On the other hand, as we have shown, increasing the content of the Higgs sector of a theory can easily spoil or make difficult the perturbative unification of the gauge couplings, unless the the new Higgses lie at the GUT scale.

Finally, we want to stress that the gauge coupling unification scale could be different from the grand unification scale, as recently proposed in [32]. In this model, the proton can naturally become almost stable if the grand unification scale is big enough compared to the scale where the gauge couplings meet.

\section{Conclusions}

In this paper, we have argued that the particle content of any extension of the Standard Model or the MSSM is tightly constrained if gauge coupling unification, perturbativity, and a GUT scale above the generic scale of proton decay, $M_{B B}^{\min } \sim 10^{16} \mathrm{GeV}$, are demanded. For example, we have demonstrated that in many left-right symmetric and Pati-Salam models, it is impossible to fulfil all three requirements simultaneously (cf. fig. 22 : Either, one has to live with unification at lower scales and invent a mechanism to circumvent proton decay bounds, or one has to extend the particle content further in order to push $M_{\mathrm{GUT}}$ higher, but at the price of losing perturbativity and thus predictivity. We have then generalized our observations to a wider class of SM or MSSM extensions, and have examined the constraints 
that perturbativity imposes on the scale of new physics and on its contribution to the $\beta$ function coefficients (cf. figs. 5 and [6). Since extra matter particles will always increase the values of the running gauge coupling constants at high scales, our constraints favour extensions of the Standard Model that require not too many new particles at low scales. In other words, the problems of the SM should be solved in a rather economic way. In our opinion, this is another hint in favour of the MSSM with $M_{\text {SUSY }} \lesssim 1 \mathrm{TeV}$.

We have finally discussed how the perturbativity constraints can be circumvented either by simply accepting non-perturbativity, by designing models with partial unification, by introducing extra dimensions, or by aiming for Grand Unification at low to intermediate scales. In the latter case, special measures have to be taken to forbid or suppress proton decay.

\section{Acknowledgments}

We would like to thank J. W. F. Valle for very interesting and useful discussions. This work was in part supported by the Sonderforschungsbereich TR 27 'Neutrinos and Beyond' der Deutschen Forschungsgemeinschaft. JK would like to acknowledge support from the Studienstiftung des Deutschen Volkes.

\section{A Analytic results for the minimal SUSY LR model}

In the following we define

$$
t_{a}=\log \left(\frac{M_{a}}{M_{Z}}\right),
$$

and firstly consider the minimal SUSY LR model detailed in ref. [14]. After solving the 1-loop RGEs for the 4 gauge couplings, we find that

$$
\alpha_{3}^{-1}\left(M_{Z}\right)=\frac{1}{4}\left(9 \alpha_{2}^{-1}\left(M_{Z}\right)-5 \alpha_{1}^{-1}\left(M_{Z}\right)\right)+\frac{1}{8 \pi}\left(30 t_{L R}-9 t_{\mathrm{SUSY}}\right) .
$$

In this model the unification scale, $M_{\mathrm{GUT}}$, is given by

$$
t_{\mathrm{GUT}}=\frac{2 \pi}{135}\left(10 \alpha_{1}^{-1}\left(M_{Z}\right)-3 \alpha_{2}^{-1}\left(M_{Z}\right)-7 \alpha_{3}^{-1}\left(M_{Z}\right)\right)+\frac{71}{270} t_{\mathrm{SUSY}} .
$$

\section{B Analytic results for the non-minimal LR Model with interme- diate $B-L$ scale}

We consider the non-minimal LR model of refs. [15, 16] and assume the hierarchy $t_{Z}<$ $t_{\mathrm{SUSY}}<t_{B L}<t_{L R}<t_{\mathrm{GUT}}$, where $M_{\mathrm{GUT}}$ is the unification scale. In addition we assume that $M_{B-L}^{2} / M_{L R}>M_{\mathrm{SUSY}}$, thus ensuring that the light $\mathrm{SU}(2)_{L}$ triplet in this model has a mass above the SUSY breaking scale (to be consistent with the results of [16]).

After solving the 1-loop RGEs for the 4 gauge couplings, we find that

$$
\alpha_{3}^{-1}\left(M_{Z}\right)=\frac{1}{32}\left(87 \alpha_{2}^{-1}\left(M_{Z}\right)-55 \alpha_{1}^{-1}\left(M_{Z}\right)\right)+\frac{1}{64 \pi}\left(216 t_{B L}-36 t_{L R}+97 t_{\mathrm{SUSY}}\right) .
$$


Thus, given values of $M_{\mathrm{SUSY}}$ and $M_{L R}$, it is possible to calculate the $M_{B-L}$ needed for successful unification, assuming the measured values of the gauge couplings at $M_{Z}$. If this $M_{B-L}$ is self-consistent with the assumptions above, then unification is possible for the specific choices of $M_{\mathrm{SUSY}}$ and $M_{L R}$. In this case we find,

$$
\begin{aligned}
t_{B L} & =\frac{1}{216}\left\{2 \pi\left(55 \alpha_{1}^{-1}\left(M_{Z}\right)-87 \alpha_{2}^{-1}\left(M_{Z}\right)+32 \alpha_{3}^{-1}\left(M_{Z}\right)\right)+36 t_{L R}-97 t_{\mathrm{SUSY}}\right\} \\
t_{\mathrm{GUT}} & =\frac{1}{864}\left\{32 \pi\left(5 \alpha_{1}^{-1}\left(M_{Z}\right)-3 \alpha_{2}^{-1}\left(M_{Z}\right)-2 \alpha_{3}^{-1}\left(M_{Z}\right)\right)+288 t_{L R}-128 t_{\mathrm{SUSY}}\right\},
\end{aligned}
$$

where the value of $\alpha_{\mathrm{GUT}}$ at the unification scale is

$$
\begin{aligned}
\alpha_{\mathrm{GUT}}^{-1}\left(M_{\mathrm{GUT}}\right)=\frac{1}{72 \pi}\{ & 4 \pi\left(5 \alpha_{1}^{-1}\left(M_{Z}\right)-3 \pi \alpha_{2}^{-1}\left(M_{Z}\right)+16 \pi \alpha_{3}^{-1}\left(M_{Z}\right)\right) \\
& \left.+36 t_{L R}+128 t_{\mathrm{SUSY}}\right\} .
\end{aligned}
$$

It is also possible to find solutions in this model with gauge coupling unification but with $M_{B-L}$ such that $M_{B-L}^{2} / M_{L R}<M_{\mathrm{SUSY}}$. In this case we assume that the light $\mathrm{SU}(2)_{L}$ triplet acquires a mass at the SUSY breaking scale $M_{\text {SUSY }}$. This leads to different unification conditions and the prediction for $\alpha_{3}^{-1}\left(M_{Z}\right)$ is now

$$
\alpha_{3}^{-1}\left(M_{Z}\right)=\frac{1}{32}\left(87 \alpha_{2}^{-1}\left(M_{Z}\right)-55 \alpha_{1}^{-1}\left(M_{Z}\right)\right)+\frac{1}{64 \pi}\left(138 t_{L R}-132 t_{B L}+271 t_{\mathrm{SUSY}}\right) .
$$

As before, one can now predict $M_{B-L}$ and assuming that the condition $t_{\mathrm{SUSY}}<t_{B L}<t_{L R}$ is met then successful unification is possible with

$$
\begin{aligned}
t_{B L}=\frac{1}{132}\left\{2 \pi\left(55 \alpha_{1}^{-1}\left(M_{Z}\right)-87 \alpha_{2}^{-1}\left(M_{Z}\right)+32 \alpha_{3}^{-1}\left(M_{Z}\right)\right)\right. \\
\left.\left.+138 t_{L R}+271 t_{\mathrm{SUSY}}\right)\right\}, \\
t_{\mathrm{GUT}}=\frac{1}{1056}\left\{192 \pi\left(\alpha_{2}^{-1}\left(M_{Z}\right)-\alpha_{3}^{-1}\left(M_{Z}\right)\right)+480 t_{L R}+208 t_{\mathrm{SUSY}}\right\}, \\
\alpha_{\mathrm{GUT}}^{-1}\left(M_{\mathrm{GUT}}\right)=\frac{1}{88 \pi}\left\{8 \pi\left(3 \alpha_{2}^{-1}\left(M_{Z}\right)+8 \alpha_{3}^{-1}\left(M_{Z}\right)\right)+60 t_{L R}+202 t_{\mathrm{SUSY}}\right\} .
\end{aligned}
$$

\section{Analytic results for the minimal Pati-Salam model}

In the minimal Pati-Salam model of ref. [25], we first assume that $M_{L R}^{2} / M_{P S}>M_{\mathrm{SUSY}}$, thus ensuring the colour octet remains heavier than $M_{\text {SUSY }}$. After solving the RGEs, the following analytic expressions are found

$$
\alpha_{3}^{-1}\left(M_{Z}\right)=\frac{1}{2}\left(5 \alpha_{1}^{-1}\left(M_{Z}\right)-3 \alpha_{2}^{-1}\left(M_{Z}\right)\right)+\frac{1}{\pi}\left(4 t_{\mathrm{SUSY}}-12 t_{L R}-9 t_{P S}\right),
$$

where $M_{P S}$ is the Pati-Salam symmetry breaking scale. Unification occurs at

$$
t_{\mathrm{GUT}}=\frac{5 \pi}{6}\left(\alpha_{2}^{-1}\left(M_{Z}\right)-\alpha_{1}^{-1}\left(M_{Z}\right)\right)+t_{L R}+\frac{13}{3} t_{P S}-\frac{47}{36} t_{\mathrm{SUSY}} .
$$


In cases where $M_{L R}^{2} / M_{P S}<M_{\mathrm{SUSY}}$, we instead assume that the colour octet has a mass of $M_{\mathrm{SUSY}}$, and the following analytic expression for $\alpha_{3}^{-1}\left(M_{Z}\right)$ is found

$$
\alpha_{3}^{-1}\left(M_{Z}\right)=\frac{1}{2}\left(5 \alpha_{1}^{-1}\left(M_{Z}\right)-3 \alpha_{2}^{-1}\left(M_{Z}\right)\right)+\frac{1}{2 \pi}\left(5 t_{\mathrm{SUSY}}-6 t_{L R}-21 t_{P S}\right) .
$$

The expression for the unification scale however remains unchanged.

[1] U. Amaldi, W. de Boer, and H. Furstenau, Phys. Lett. B260, 447 (1991).

[2] U. Amaldi, W. de Boer, P. H. Frampton, H. Furstenau, and J. T. Liu, Phys. Lett. B281, 374 (1992).

[3] M. Lindner and M. Weiser, Phys. Lett. B383, 405 (1996), hep-ph/9605353.

[4] L. Calibbi, L. Ferretti, A. Romanino, and R. Ziegler, Phys. Lett. B672, 152 (2009), 0812.0342 .

[5] N. T. Shaban and W. J. Stirling, Phys. Lett. B291, 281 (1992).

[6] A. Perez-Lorenzana and R. N. Mohapatra, Nucl. Phys. B559, 255 (1999), hep-ph/9904504.

[7] A. Perez-Lorenzana, W. A. Ponce, and A. Zepeda, Phys. Rev. D59, 116004 (1999), hep-ph/9812401.

[8] A. Aranda, J. L. Diaz-Cruz, and A. D. Rojas (2009), 0907.4552.

[9] D. R. T. Jones, Phys. Rev. D25, 581 (1982).

[10] R. N. Mohapatra, F. E. Paige, and D. P. Sidhu, Phys. Rev. D17, 2462 (1978).

[11] R. N. Mohapatra and G. Senjanovic, Phys. Rev. Lett. 44, 912 (1980).

[12] R. N. Mohapatra and G. Senjanovic, Phys. Rev. D23, 165 (1981).

[13] N. G. Deshpande, J. F. Gunion, B. Kayser, and F. I. Olness, Phys. Rev. D44, 837 (1991).

[14] K. S. Babu and R. N. Mohapatra, Phys. Lett. B668, 404 (2008), 0807.0481.

[15] C. S. Aulakh, K. Benakli, and G. Senjanovic, Phys. Rev. Lett. 79, 2188 (1997), hep-ph/9703434.

[16] C. S. Aulakh, A. Melfo, A. Rasin, and G. Senjanovic, Phys. Rev. D58, 115007 (1998), hep-ph/9712551.

[17] C. Amsler et al. (Particle Data Group), Phys. Lett. B667, 1 (2008).

[18] H. Murayama and A. Pierce, Phys. Rev. D65, 055009 (2002), hep-ph/0108104.

[19] Y. Nomura, D. Tucker-Smith, and N. Weiner, Nucl. Phys. B613, 147 (2001), hep-ph/0104041.

[20] R. Dermisek and A. Mafi, Phys. Rev. D65, 055002 (2002), hep-ph/0108139.

[21] K. S. Babu and S. M. Barr, Phys. Rev. D48, 5354 (1993), hep-ph/9306242.

[22] K. S. Babu and S. M. Barr, Phys. Rev. D65, 095009 (2002), hep-ph/0201130.

[23] B. Bajc, A. Melfo, G. Senjanovic, and F. Vissani, Phys. Rev. D70, 035007 (2004), hep-ph/0402122.

[24] J. C. Pati and A. Salam, Phys. Rev. D10, 275 (1974).

[25] A. Melfo and G. Senjanovic, Phys. Rev. D68, 035013 (2003), hep-ph/0302216.

[26] M. Maniatis (2009), 0906.0777.

[27] C. Csaki, G. Marandella, Y. Shirman, and A. Strumia, Phys. Rev. D73, 035006 (2006), hep-ph/0510294. 
[28] H. Nishino et al. (Super-Kamiokande), Phys. Rev. Lett. 102, 141801 (2009), 0903.0676 .

[29] K. Kobayashi et al. (Super-Kamiokande), Phys. Rev. D72, 052007 (2005), hep-ex/0502026.

[30] K.-S. Choi, Phys. Lett. B668, 392 (2008), 0807.2766.

[31] R. N. Mohapatra, Phys. Rev. Lett. 43, 893 (1979).

[32] Y. Kawamura (2009), 0906.3773. 